\title{
Restoration of Halftoned Color-Quantized Images Using Linear Estimator
}

\author{
Yik-Hing Fung and Yuk-Hee Chan \\ Centre for Multimedia Signal Processing \\ Department of Electronic and Information Engineering \\ The Hong Kong Polytechnic University, Hong Kong
}

\begin{abstract}
Restoration of color-quantized images in which error diffusion is involved is rarely addressed in the literature. Conventional restoration algorithms were generally proposed for handling other noise models and hence they are not effective in restoring color-quantized images. In this paper, we tackle this problem by making use of the best linear estimation. It is shown by the simulation results that the algorithm can improve the quality of colorquantized images to a certain extent.
\end{abstract}

\section{INTRODUCTION}

Color quantization is a process of reducing the number of colors in a digital color image by replacing them with a representative color selected from a palette[1-4]. This palette is usually image dependent and it can be of any arbitrary size and contain arbitrary colors. When color quantization is performed, certain types of degradation are introduced due to the limited colors used to produce the output image. By using digital halftoning $[5,6]$, these defects can be eliminated by making use of the fact that human eyes act as spatial low-pass filters. At the moment, the most popular halftoning method is error diffusion and several well-known error diffusion filters such as Floyd-Steinberg filter[7], Jarvis-Judice-Ninke filter[8] and Stucki filter[9] are generally used to solve the problem.

Figure 1 shows the functional block diagram of a system which performs color quantization with error diffusion. Hereafter, we refer to the output of this system as a halftoned color-quantized (HCQ)image.

There is degradation when color quantization is applied to the original full-color image. In some applications, image restoration is necessary to recover the original image from its color-quantized version. This is especially true when the color-quantized version is required to be further processed or compressed.

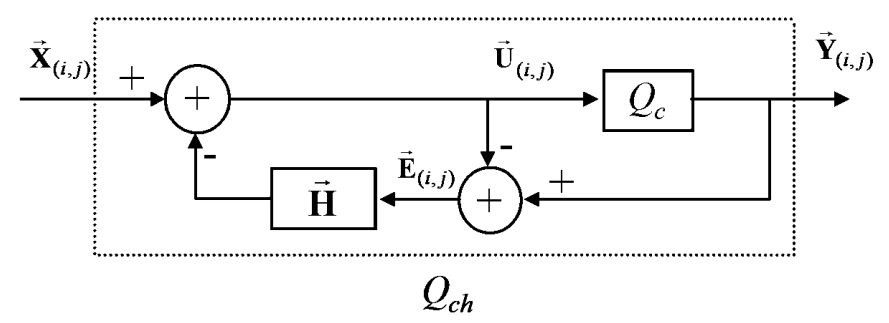

Figure 1. Color quantization with error diffusion
However, though there are a lot of reported works on the restoration of noisy and blurred color images[10-18], little effort has been seen in the literature for restoring halftoned color-quantized images. Obviously, the degradation models of the two cases are completely different and hence direct adoption of conventional restoration algorithms does not work effectively. Recently, a restoration algorithm for handling color-quantized images has been proposed, but it does not take account of the case when halftoning is involved in the quantization process[19].

In printing applications, color images are decomposed into 3 or 4 color components (CMY or CMYK). Each color plane is then considered as an individual gray scale image and they are separately halftoned with a conventional error diffusion algorithm. Accordingly, to restore the color prints into its original, one can process different color planes separately by inverse halftoning.

Recently, Mese proposed a LUT(Look-Up Table)based approach[12] for inverse halftoning and it was shown to be a universal and efficient approach as it does not require any explicit assumption of the image model exploited in the derivation of the algorithm and its realization can be very fast.

To determine the inverse halftone value of a particular pixel, Mese's algorithm[12] inspects the intensity distribution of the pixels in the neighborhood of the pixel of interest and, based upon the intensity distribution, assigns a contone value from a precomputed LUT. This significantly reduces the computation effort of inverse halftoning.

In inverse halftoning, the input image is a bi-level halftone. The number of input patterns fed into the LUT is expected to be very limited and hence it is possible to construct a LUT of a reasonably small size. In particular, for a 16-pixel neighborhood, it needs a LUT of only $2^{16}=64 \mathrm{~K}$ bytes. Besides, as there are only a few involved input patterns, just a few of training images are already good enough to provide sufficient number of training samples to construct the LUT.

Even so, it is possible that there are some null entries in the constructed LUT as some patterns do not exist in the training images. When this happens, the best linear estimator is derived with the available LUT entries and used to estimate the contone values of the corresponding patterns.

By using this LUT-based approach, inverse halftoning can be done efficiently to provide a contone image of good 
image quality. However, this LUT-based approach does not work when it is exploited in restoring halftoned colorquantized images. It is because, for handling a colorquantized image with 256 colors, it requires a LUT of size $256^{16}=3.4 \times 10^{38}$ bytes for a 16 -pixel support. The memory requirement of the large table makes the LUT-based approach become not practical when the number of pixel intensity levels of the input image is large. Hence, table look-up is not a suitable restoration approach for restoring halftoned color-quantized images.

Besides, color quantization is actually a vector quantization instead of a bi-level uniform scalar quantization as in the case of binary halftoning. By considering this, restoring color prints generated for printing applications is only a special case of the problem concerned in this paper. In particular, it is equivalent to the case that a palette $\{(\mathrm{C}, \mathrm{M}, \mathrm{Y}) \mid \mathrm{C}, \mathrm{M}, \mathrm{Y}=0,1\}$, where 0 and 1 denote the minimum and maximum intensity values respectively, is used in color quantization.

In this paper, we modify Mese's algorithm[12] to restore halftoned color-quantized images with a linear prediction filter. Instead of constructing a LUT for restoration, we use the linear prediction filter with best linear estimator to estimate the color value for a given color pattern. By doing so, a LUT of large size can be eliminated.

The organization of this paper is as follows. Section II provides a system overview of color quantization in which error diffusion is involved. Section III presents a restoration method for restoring halftoned color-quantized images. Section IV provides some simulation results to evaluate the performance of the algorithm. Finally, a concluding remark is given.

\section{OVERVIEW OF CQ WITH ERROR DifFUSION}

Full-color image $\mathbf{X}$ generally consists of three 8-bit color planes, say, $\mathbf{X}_{\mathrm{r}}, \mathbf{X}_{\mathrm{g}}$ and $\mathbf{X}_{\mathrm{b}}$, which represents the red, the green and the blue color planes of the image respectively. A pixel is then a vector represented as $\overrightarrow{\mathbf{X}}_{(i, j)}=\left(\mathbf{X}_{(i, j) r}, \mathbf{X}_{(i, j) g}, \mathbf{X}_{(i, j) b}\right)$, where $\mathbf{X}_{(i, j) c} \in[0,1]$ is the intensity value of the $c^{\text {th }}$ color component of the $(i, j)^{\text {th }}$ pixel. Here, we assume that the image is of size $N \times N$ and the maximum and the minimum intensity values of a pixel are, respectively, 1 and 0 . Figure 1 shows the system which performs color quantization with error diffusion. The input image $\mathbf{X}$ is scanned in a row-by-row fashion from pixel $(1,1)$ to pixel $(N, N)$ and processed as follows to produce the encoded image $\mathbf{Y}$.

$$
\begin{aligned}
& \mathbf{U}_{(i, j) c}=\mathbf{X}_{(i, j) c}-\sum_{(k, l) \in S} \mathbf{H}_{(k, l) c} \mathbf{E}_{(i-k, j-l) c} \\
& \overrightarrow{\mathbf{Y}}_{(i, j)}=Q_{c}\left[\overrightarrow{\mathbf{U}}_{(i, j)}\right] \\
& \overrightarrow{\mathbf{E}}_{(i, j)}=\overrightarrow{\mathbf{Y}}_{(i, j)}-\overrightarrow{\mathbf{U}}_{(i, j)}=Q_{c}\left[\overrightarrow{\mathbf{U}}_{(i, j)}\right]-\overrightarrow{\mathbf{U}}_{(i, j)}
\end{aligned}
$$$$
\text { and }
$$

where $\overrightarrow{\mathbf{U}}_{(i, j)}$ is a state vector of the system, $\overrightarrow{\mathbf{E}}_{(i, j)}$ is the quantization error of the pixel at position $(i, j)$ and $\mathbf{H}_{(k, l) c}$ is a coefficient of the error diffusion filter for the $c^{\text {th }}$ color component. $S$ is the casual support region of $\mathbf{H}_{(k, l) c}$. The operator $Q_{c}[\bullet]$ performs a $3 \mathrm{D}$ vector quantization. Specifically, the 3D vector $\overrightarrow{\mathbf{U}}_{(i, j)}$ is compared with a set of representative color vectors stored in a previously generated color palette $C=\left\{\hat{\mathbf{v}}_{i}: i=1,2, \ldots N_{c}\right\}$, where $\hat{\mathbf{v}}_{i}=\left(v_{i, r}, v_{i, g}, v_{i, b}\right)$. The best-matched vector in the palette is selected based on the minimum Euclidean distance criterion. In other words, a state vector $\overrightarrow{\mathbf{U}}_{(i, j)}$ is represented by color $\hat{\mathbf{v}}_{k}$ if and only if $\left\|\overrightarrow{\mathbf{U}}_{(i, j)}-\hat{\mathbf{v}}_{k}\right\| \leq\left\|\overrightarrow{\mathbf{U}}_{(i, j)}-\hat{\mathbf{v}}_{l}\right\|$ for all $l=1,2 \ldots N_{c}$. Once the best-matched vector is selected from the color palette, its index is recorded and the quantization error $\overrightarrow{\mathbf{E}}_{(i, j)}=\hat{\mathbf{v}}_{k}-\overrightarrow{\mathbf{U}}_{(i, j)}$ is diffused to pixel $(i, j)$ 's neighborhood with eqn. (1). To handle the boundary pixels, $\overrightarrow{\mathbf{E}}_{(i, j)}$ is defined to be zero when $(i, j)$ falls outside the image. After the scanning is finished, the recorded indices can be used in the future to reconstruct the halftoned color-quantized image with the same color palette.

\section{RESTORATION OF HCQ IMAGES}

This section presents a method for restoring halftoned color-quantized images. It is basically an extension of Mese's work[12]. In this method, the original color of a halftoned color-quantized pixel is estimated with the colors of its neighboring pixels. Since a large look-up table is not practical for restoring halftoned color-quantized images, the LUT used in Mese's algorithm [12] is omitted and a minimum least-squares linear estimator is used to achieve the goal. To obtain the linear estimator, a set of standard color training images and its halftoned color-quantized versions are used to form a training set $R$.

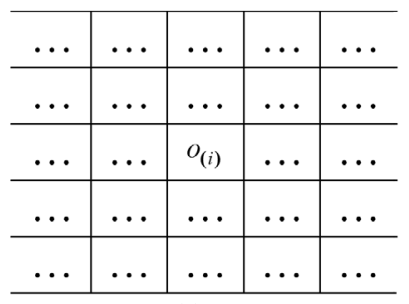

(a)

\begin{tabular}{c|c|c|c|c}
\hline$\ldots$ & $p_{(i, 0)}$ & $p_{(i, 1)}$ & $p_{(i, 2)}$ & $\ldots$ \\
\hline$p_{(i, 3)}$ & $p_{(i, 4)}$ & $p_{(i, 5)}$ & $p_{(i, 6)}$ & $\ldots$ \\
\hline$p_{(i, 7)}$ & $p_{(i, 8)}$ & $p_{(i, 9)}$ & $p_{(i, 10)}$ & $p_{(i, 11)}$ \\
\hline$\ldots$ & $p_{(i, 12)}$ & $p_{(i, 13)}$ & $p_{(i, 14)}$ & $\ldots$ \\
\hline$\ldots$ & $\ldots$ & $p_{(i, 15)}$ & $\ldots$ & $\ldots$ \\
\hline
\end{tabular}

(b)
Figure 2. (a) A pixel of interest in the original color image and (b) the context of the pixel's corresponding halftoned color-quantized pattern in the halftoned color-quantized image.

Each element of the training set contains two components. One of them is a scalar which represents a color intensity value of a particular pixel in the original fullcolor images. This particular pixel is the pixel of interest $\left(o_{(i)}\right.$ in Figure 2a) and the color intensity values of the ordered pixels in this pixel's associated neighborhood in the 
corresponding halftoned color-quantized image form a vector. Figure $2 \mathrm{~b}$ shows the neighborhood associated with this pixel. Note that the centers of Figure $2 \mathrm{a}$ and Figure $2 \mathrm{~b}$ align with each other.

Let $\vec{p}_{i}=\left(p_{(i, 0)}, p_{(i, 1)}, \ldots, p_{(i, N-1)}\right)$ be the vector associated with $o_{(i)}$, where $N$ is the size of the neighborhood in terms of number of pixels ( $N=16$ in our case). Each pair of $\vec{p}_{i}$ and $o_{(i)}$ forms an element of the training set. All elements in the training set $R$ are used to derive the best linear estimator for estimating $o_{(i)}$ with $\vec{p}_{i}$. In particular, the estimator can be obtained by deriving the $\overrightarrow{\mathbf{w}}$ which minimizes the following objective function.

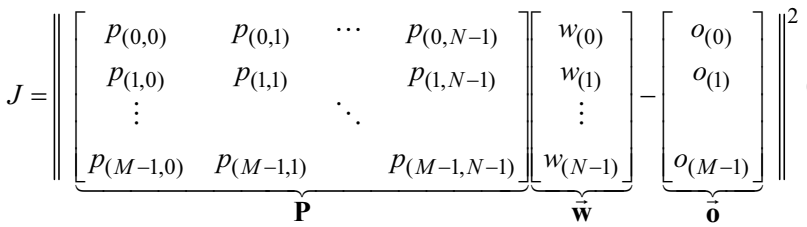

where $M$ is the total number of elements in the training set $R$. The solution is given as

$$
\overrightarrow{\mathbf{w}}=\left(\mathbf{P}^{T} \mathbf{P}\right)^{-1} \mathbf{P}^{T} \overrightarrow{\mathbf{o}}
$$

In the restoration, the observed image, say $\mathbf{Y}$, is scanned in a raster scanning fashion. For each pixel, a local region which matches the template used in the training phase is extracted to form a vector $\vec{y}=\left(y_{(0)}, y_{(1)}, \ldots, y_{(N-1)}\right)$. The true color value of pixel $y_{(9)}$, say $x$, is then estimated with $\vec{y}$ and the best linear estimator $\overrightarrow{\mathbf{w}}$ as follows.

$$
x=\vec{y} \overrightarrow{\mathbf{w}}
$$

After $x$ is obtained, it is rounded to the nearest displayable color level for display.

\section{Simulation AND COMPARATIVE STUdY}

To evaluate the performance, simulation was carried out on a set of halftoned color-quantized images. In our simulation, a number of de facto standard 24-bit full-color images including "Lenna", "Baboon", "Peppers", "Fruits", "Couple", "Girl”, "Parrots", "Pool”, "Caps" and "Window" were used. Each of them is of size 256x256. For each image, a set of color palettes were generated with median cut algorithm[2] and their sizes varied from 16 to 128. Each testing image was then color-quantized with a palette to produce $\mathbf{Y}$ 's. In color quantization, halftoning is performed with error diffusion and the Floyd-Steinberg diffusion filter[7] was used. The proposed restoration algorithm was applied to restore the halftoned colorquantized images (i.e. $\mathbf{Y}$ ). In the training phase of the proposed algorithm, "Baboon", "Melon", "House", "Lenna" and their corresponding halftoned color-quantized outputs were used to train the best linear estimator.

For comparison, some other restoration algorithms were also evaluated. Among them, Galatsanos's algorithm [13] is based on the constrained least square approach and Hunt's algorithm[14] is based on Wiener filtering. There are two algorithms presented in Altunbasak's work[15]. The first one makes use of the correlation among the color components of a pixel while the other one does not. They are, respectively, referred to as KL and IND in [15]. All the aforementioned algorithms were originally proposed for handling noisy blurred color images. Fung's algorithm [19] adopts a POCS-based approach. Unlike [13], [14] and [15], it was proposed for restoring color-quantized images. However, it does not take the halftoning process into account during the restoration.

In realizing Galatsanos's algorithm[13], the noise power of each channel was estimated with the original fullcolor image. In realizing Hunt's algorithm[14], three separate Wiener filters were used in three different channels and, during the design of the filters, the noise spectrum of each channel was estimated with the original full-color image. Similarly, the original full-color image was used to estimate the power spectra of different channels in realizing Altunbasak's algorithms[15]. In a practical situation, no original image is available and hence all must be estimated from the degraded image.

The performance of the algorithms was evaluated as follows. For each color-quantized image $\mathbf{Y}$ generated with a particular testing image $\mathbf{X}$, a restored output $\hat{\mathbf{X}}$ was obtained with a particular algorithm. The restoration result $\hat{\mathbf{X}}$ is evaluated in terms of SNR improvement and the CIELAB color difference ( $\Delta E$ ) metric[20]. The figures shown in Table 1 show the average values of the evaluation results of all standard testing images.

From Table 1a, one can see that the performance of the proposed algorithm is better as compared with the others. Here, the SNR improvement is defined as $\mathrm{SNRI}=10 \log \left(\|\mathbf{X}-\mathbf{Y}\|^{2} /\|\mathbf{X}-\hat{\mathbf{X}}\|^{2}\right)$.

It is well accepted that color errors are visually detectable when $\Delta E>3[15,21]$. Table $1 \mathrm{~b}$ shows the average of the $\Delta E$ values of all pixels in all restoration outputs and Table 1c shows the average of the percentage of pixels whose color error is visually undetectable in a restoration output. Again, one can see that the proposed algorithm is superior to the others.

\section{CONCLUDING REMARKS}

In this paper we introduce a restoration algorithm for restoring halftoned color-quantized images. This algorithm makes use of a set of training images to derive the best linear estimator for restoration. Simulation results demonstrated that the presented algorithm could achieve a better restoration performance in terms of both SNR and CIELAB color difference ( $\Delta E)$ metric as compared with some other restoration algorithms.

\section{ACKNOWLEDGEMENT}

This work was supported by a grant from the Research Grants Council of the Hong Kong Special Administrative Region (PolyU 5205/04E). 


\begin{tabular}{|c|c|c|c|c|c|c|c|c|}
\hline & \multirow{3}{*}{$\begin{array}{l}\text { Palette } \\
\text { size }\end{array}$} & \multirow{3}{*}{$\begin{array}{l}\text { Observed } \\
(\mathbf{Y})\end{array}$} & \multicolumn{6}{|c|}{ Restored ( $\left.\mathbf{X}^{\prime}\right)$} \\
\hline & & & \multirow[b]{2}{*}{ Proposed } & \multirow{3}{*}[18]{} & \multirow{2}{*}{$\begin{array}{l}{[14]} \\
\text { IND }\end{array}$} & \multirow{2}{*}{$\begin{array}{c}{[14]} \\
\mathrm{KL}\end{array}$} & \multirow[b]{2}{*}[12]{} & \multirow[b]{2}{*}[13]{} \\
\hline & & & & & & & & \\
\hline \multirow{5}{*}{$\mathrm{a}$} & & \multicolumn{6}{|c|}{ Average of (SNR Improvement (dB)) } & \\
\hline & 128 & - & 5.29 & 4.69 & 5.17 & 4.85 & 2.23 & 4.05 \\
\hline & 64 & - & 6.63 & 4.51 & 5.79 & 5.45 & 3.13 & 4.64 \\
\hline & 32 & - & 7.71 & 3.90 & 5.88 & 5.64 & 3.95 & 4.87 \\
\hline & 16 & - & 7.63 & 3.48 & 5.45 & 5.34 & 3.59 & 4.63 \\
\hline \multirow{5}{*}{$\mathrm{b}$} & & \multicolumn{7}{|c|}{ Average of (Average of CIELAB difference $\Delta E$ ) } \\
\hline & 128 & 5.82 & 4.40 & 4.18 & 4.24 & 4.35 & 5.22 & 4.65 \\
\hline & 64 & 7.29 & 5.17 & 5.42 & 5.17 & 5.35 & 6.29 & 5.75 \\
\hline & 32 & 8.95 & 6.27 & 7.03 & 6.47 & 6.72 & 7.70 & 7.12 \\
\hline & 16 & 11.10 & 8.08 & 9.22 & 8.40 & 8.61 & 10.03 & 8.96 \\
\hline \multirow{5}{*}{ c } & & \multicolumn{7}{|c|}{ Average of (\% of pixels whose CIELAB $\Delta E<3$ ) } \\
\hline & 128 & 32.29 & 45.02 & 48.86 & 47.25 & 45.94 & 36.98 & 42.16 \\
\hline & 64 & 24.17 & 37.69 & 36.99 & 37.01 & 35.57 & 29.84 & 31.77 \\
\hline & 32 & 15.25 & 28.89 & 25.66 & 26.33 & 24.92 & 21.80 & 21.54 \\
\hline & 16 & 8.43 & 19.18 & 16.05 & 17.36 & 16.55 & 12.46 & 14.77 \\
\hline
\end{tabular}

Table 1. Average performance of various algorithms in restoring halftoned color-quantized images in various aspects

\section{REFERENCE}

[1] M.T. Orchard and C.A. Bouman, "Color quantization of images," IEEE Trans. SP, Vol.39, pp.2677-2690, 1991.

[2] P. Heckbert, "Color image quantization for frame buffer displays," Comput. Graph., Vol.16, No.4, pp. 297-307, 1982.

[3] S.S. Dixit, "Quantization of color images for display/printing on limited color output devices," Comput.Graph., Vol. 15, No. 4, pp. 561$567,1991$.

[4] $\mathrm{X}$. Wu, "Color quantization by dynamic programming and principal analysis," ACM Trans. Graph., Vol. 11, No. 4, pp.384-372, 1992.

[5] R. Ulichney, Digital Halftoning. Cambridge, MA:MIT Press, 1987.

[6] R.S. Gentile, E.Walowit, and J.P. Allebach, "Quantization and multilevel halftoning of color images for near original image quality," Proc. SPIE, Vol.1249, pp. 249-259, 1990.

[7] R. W. Floyd and L. Steinberg, "An Adaptive Algorithm for Spatial Grayscale," Proceedings of the Society for Information Display, Vol.17, No.2, pp.75-77, 1976

[8] J. F. Jarvis, C. N. Judice and W. H. Ninke, "A Survey of Techniques for the Display of Continuous Tone Pictures on Bi-level Displays," Computer Graphics and Image Processing, 5, pp.13-40, 1976

[9] P. Stucki, "MECCA - a multiple error correcting computation algorithm for bi-level image hard copy reproduction," Research report RZ1060, IBM Research Laboratory, Zurich, Switzerland, 1981.

[10] M. Barni, V. Cappellini and L. Mirri, "Multichannel filtering for color image restoration," Proc., IEEE ICIP'2000, 2000, Vol.1, pp. 529-532.

[11] G.Angelopoulos and I. Pitas, "Multichannel wiener filters in color image restoration," IEEE Trans. CASVT, Vol.4, pp.83-87, 1994.

[12] M. Mese and P. P. Vaidyanathan. "Look-Up Table (LUT) Method for Inverse Halftoning," IEEE Trans. On Image Processing, Vol.10, No.10, pp.1566-1578, Oct 2001

[13] N.P. Galatsanos, A.K. Katsaggelos, R.T. Chin, and A.D. Hillery, "Least squares restoration of multichannel images," IEEE Trans. SP, Vol.39, pp.2222-2236, 1991.

[14] B.R. Hunt and O.Kubler, "Karhunen-Loeve multispectral image restoration, Part 1: Theory," IEEE Trans. ASSP, Vol.32, pp.592-600, 1984.

[15] H.Altunbasak and H.J. Trussell, "Colorimetric restoration of digital images,” IEEE Trans. IP, Vol.10, pp.393-402, 2001.

[16] K.J. Boo and N.K. Bose, "Multispectral image restoration with multisensor," IEEE Trans. Geoscience and RemoteSensing, Vol.35, pp.1160-1170, 1997.

[17] N.P. Galatsanos and R.T. Chin, "Digital restoration of multichannel images," IEEE Trans. ASSP, Vol.37, pp. 415-421, 1989.
[18] N.P. Galatsanos and R.T. Chin, "Restoration of color images by multichannel Kalman filtering," IEEE Trans. SP, Vol.39, pp.22372252, 1991.

[19] Y.H. Fung and Y.H. Chan, "An Iterative Algorithm for Restoring Color-Quantized Images," Proceedings, IEEE ICIP'02, 2002, Vol.1, pp.313-316.

[20] C.I.E. (1978) Recommendations on uniform color spaces, color difference equations, psychometric color terms. Supplement No.2 to CIE publication No.15 (E.-1.3.1) 1971/(TC-1.3.).

[21] C. Connolly, T.W.W. Leung and J. Nobbs, "Colour measurement by video camera," JSDC, Vol. 111, pp. 373-375, 1995.

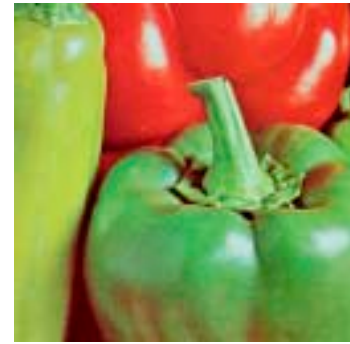

(a) Original

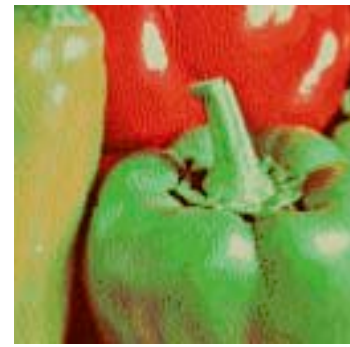

(c) Galatsanos [13]

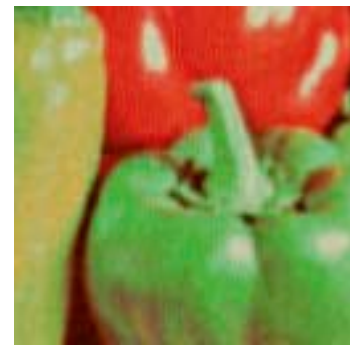

(e) IND [15]

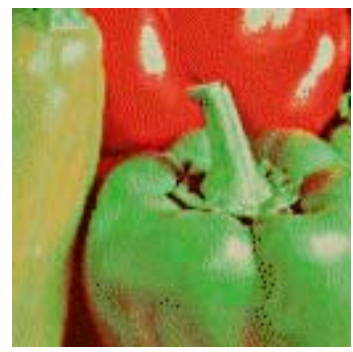

(g) Fung [19]

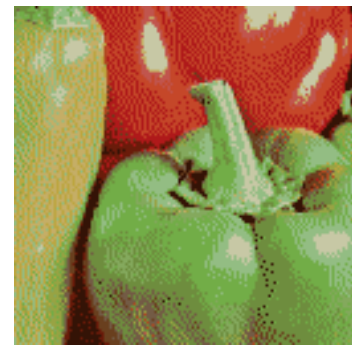

(b) halftoned color-quantized

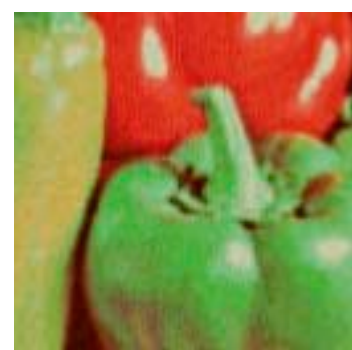

(d) Hunt [14]

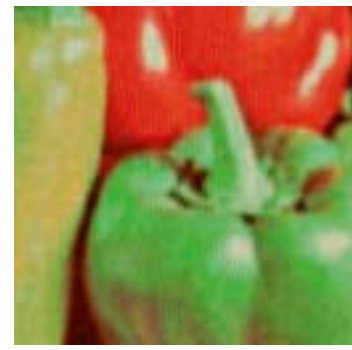

(f) KL [15]

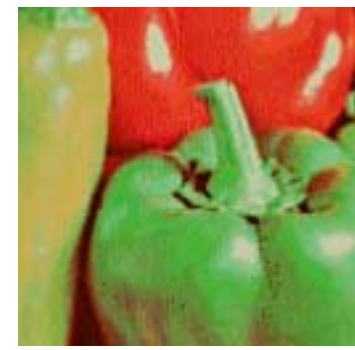

(h) Proposed
Figure 3. (a) Original, (b) halftoned color-quantized output obtained with a 16-color palette generated with median-cut algorithm[2], (c-f) restoration results of various algorithms 